Virginia Commonwealth University VCU Scholars Compass

2014

\title{
Fine structure of the red luminescence band in undoped $\mathrm{GaN}$
}

Michael A. Reshchikov

Virginia Commonwealth University, mreshchi@vcu.edu

A. Usikov

Nitride Crystals, Inc.

H. Helava

Nitride Crystals, Inc.

Yu. Makarov

Saint-Petersburg National Research University of Information Technologies

Follow this and additional works at: http://scholarscompass.vcu.edu/phys_pubs

Part of the Physics Commons

Reschchikov, M.A., Usikov, A., and Helava, H., et al., Fine structure of the red luminescence band in undoped GaN. Applied Physics Letters, 104, 032103 (2014). Copyright (C 2014 AIP Publishing LLC.

\section{Downloaded from}

http://scholarscompass.vcu.edu/phys_pubs/25

This Article is brought to you for free and open access by the Dept. of Physics at VCU Scholars Compass. It has been accepted for inclusion in Physics Publications by an authorized administrator of VCU Scholars Compass. For more information, please contact libcompass@vcu.edu. 


\title{
Fine structure of the red luminescence band in undoped GaN
}

\author{
M. A. Reshchikov, ${ }^{1, a)}$ A. Usikov, ${ }^{2,3}$ H. Helava, ${ }^{2}$ and Yu. Makarov ${ }^{2}$ \\ ${ }^{1}$ Department of Physics, Virginia Commonwealth University, Richmond, Virginia 23284, USA \\ ${ }^{2}$ Nitride Crystals, Inc., 181E Industry Ct., Ste. B, Deer Park, New York 11729, USA \\ ${ }^{3}$ Saint-Petersburg National Research University of Information Technologies, Mechanics and Optics, \\ 49 Kronverkskiy Ave., 197101 Saint Petersburg, Russia
}

(Received 23 December 2013; accepted 5 January 2014; published online 21 January 2014)

\begin{abstract}
Many point defects in GaN responsible for broad photoluminescence (PL) bands remain unidentified. Their presence in thick GaN layers grown by hydride vapor phase epitaxy (HVPE) detrimentally affects the material quality and may hinder the use of $\mathrm{GaN}$ in high-power electronic devices. One of the main PL bands in HVPE-grown GaN is the red luminescence (RL) band with a maximum at $1.8 \mathrm{eV}$. We observed the fine structure of this band with a zero-phonon line (ZPL) at $2.36 \mathrm{eV}$, which may help to identify the related defect. The shift of the ZPL with excitation intensity and the temperature-related transformation of the RL band fine structure indicate that the RL band is caused by transitions from a shallow donor (at low temperature) or from the conduction band (above $50 \mathrm{~K}$ ) to an unknown deep acceptor having an energy level $1.130 \mathrm{eV}$ above the valence band. @ 2014 AIP Publishing LLC. [http://dx.doi.org/10.1063/1.4862790]
\end{abstract}

Gallium nitride has gained unprecedented attention due to its applications in light emitters (green, blue, and ultraviolet LEDs and laser diodes) and optical detectors. It is also very promising to be used in next-generation high-power/ high frequency electronics. ${ }^{1-4}$ To fabricate high-resistivity $\mathrm{GaN}$ with high breakdown voltage, the concentration of unwanted point defects must be significantly reduced. In spite of many years of research, however, point defects with deep levels in GaN are still not well understood. The identification of these defects and their description with first-principles calculations is an important and challenging problem. Theoretical calculations of defect properties in semiconductors always involve a wide range of corrections, which are necessary to remove significant errors that originate from several different sources. Then, reliable experimental data are needed for comparison with theoretical results. Most of the deep-level defects in $\mathrm{GaN}$ produce broad, structureless luminescence bands, such as the yellow luminescence (YL) band having a maximum at $2.2 \mathrm{eV} .{ }^{5}$ For such defects, it is difficult to find the position of the transition energy level and compare it with theory. The most reliable and accurate way to determine the transition energy of a deep-level defect is to find the zero-phonon line (ZPL), which is very rarely observed, especially for deep defects. In this Letter, we report on the observation of the ZPL and fine structure for the red luminescence (RL) band having a maximum at $1.8 \mathrm{eV}$ at low temperature.

Four samples were analyzed in this work and are $\sim 10 \mu$ m-thick, unintentionally doped GaN layers grown by hydride vapor phase epitaxy (HVPE) on a $c$-plane sapphire substrate. From the Hall-effect measurements at room temperature, the concentration of free electrons and the electron mobility in these samples have been found to be $3 \times 10^{17} \mathrm{~cm}^{-3}$ and $200-300 \mathrm{~cm}^{2} \mathrm{~V}^{-1} \mathrm{~s}^{-1}$, respectively. However, these data may be affected by a thin degenerate layer at GaN/sapphire interface, which is often observed in samples grown by HVPE

\footnotetext{
a)Electronic mail: mreshchi@vcu.edu
}

method. ${ }^{6,7}$ Our preliminary analysis of time-resolved photoluminescence (PL) indicates that the concentration of free electrons is about $1 \times 10^{17} \mathrm{~cm}^{-3}$ in the $100-200 \mathrm{~nm}$-thick layer below the samples surface. We expect that the dominant shallow donor in our samples is silicon in gallium site $\left(\mathrm{Si}_{\mathrm{Ga}}\right)$. Steady-state PL was excited with an unfocused He-Cd laser $(50 \mathrm{~mW}, 325 \mathrm{~nm})$, dispersed by a $1200 \mathrm{rules} / \mathrm{mm}$ grating in a $0.3 \mathrm{~m}$ monochromator and detected by a cooled photomultiplier tube. Calibrated neutral-density filters were used to attenuate the excitation power density $\left(P_{\text {exc }}\right)$ over the range $10^{-5}-0.2 \mathrm{~W} / \mathrm{cm}^{2}$. A closed-cycle optical cryostat was used for temperatures between 15 and $320 \mathrm{~K}$.

The PL spectra of the GaN samples at $18 \mathrm{~K}$ for relatively high excitation intensity are shown in Fig. 1. The inset to this figure shows the excitonic emission with the dominant line at $\sim 3.475 \mathrm{eV}$ and full width at half maximum (FWHM) of $4.0 \mathrm{meV}$, which is attributed to the annihilation of an exciton bound to a neutral shallow donor (DBE). A weaker peak at $\sim 3.480 \mathrm{eV}$ is the free exciton (FE) line, as was verified by variation of the temperature. The low-temperature PL spectrum also contains the ultraviolet luminescence (UVL) band with the ZPL at $3.26 \mathrm{eV}$, followed by two LO phonon replicas and a broad RL band with a maximum at $1.81 \mathrm{eV}$. A weak shoulder to the RL band in the range from 2.4 to $2.9 \mathrm{eV}$ is identified as the green luminescence (GL) band. The GL band has a maximum at $2.5 \mathrm{eV}$, and it is often observed in high-quality thick GaN grown by HVPE. ${ }^{5}$ It can be easily recognized in time-resolved PL measurements due to its exponential decay even at low temperatures. ${ }^{5}$

As the excitation intensity decreases, the relative contribution of the RL band increases (Fig. 2), because the exciton emission changes superlinearly with the excitation intensity. The shape and position of the RL band are independent of the excitation intensity (with accuracy of $10 \mathrm{meV}$ ), and the FWHM of the RL band is $370 \mathrm{meV}$ at $18 \mathrm{~K}$ (Fig. 3). The shape of the RL band was the same in all of the studied samples: A less steep high-energy side with an abrupt drop at $2.36 \mathrm{eV}$. The exact same shape and position of the RL band 


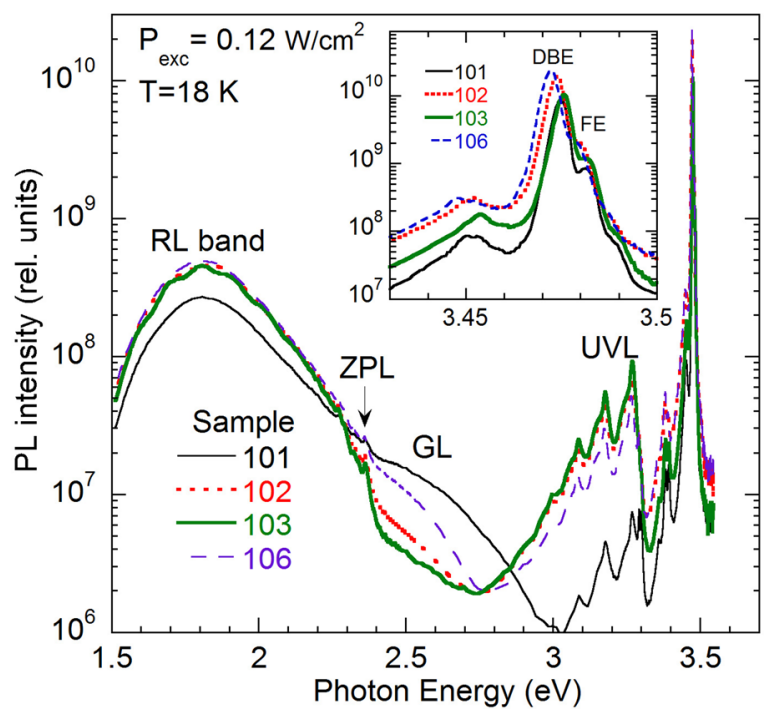

FIG. 1. PL spectra from four undoped $\mathrm{GaN}$ samples at $T=18 \mathrm{~K}$ and $P_{e x c}=0.12 \mathrm{~W} / \mathrm{cm}^{2}$. The inset shows the excitonic region in more detail. ZPL at $2.36 \mathrm{eV}$ is the zero-phonon line of the RL band.

were observed by Wang et al. ${ }^{8,9}$ in HVPE-grown GaN, yet these authors have not observed the fine structure of the RL band, which will be discussed below.

Figure 4 shows the high-energy side of the RL band in the studied samples. A sharp peak at $2.36 \mathrm{eV}$ is identified as the ZPL line of the RL band. The LO phonon replicas of this line can be clearly seen at distances of 91 and $182 \mathrm{meV}$. Two other sets of peaks with separations of 38 and $70 \mathrm{meV}$ from the ZPL (indicated with dashed lines) can be attributed to local or quasi-local phonon modes of the defect responsible for the RL band.

With increasing temperature, the UVL band transforms in a usual way: the donor-acceptor pair (DAP) peak gradually disappears, and the peak due to recombination of free electrons with the holes bound to the shallow acceptor (the eA transition) emerges at $T>30 \mathrm{~K}$ (Fig. 5(a)). Such transformation of the UVL band can be explained as follows. ${ }^{10}$

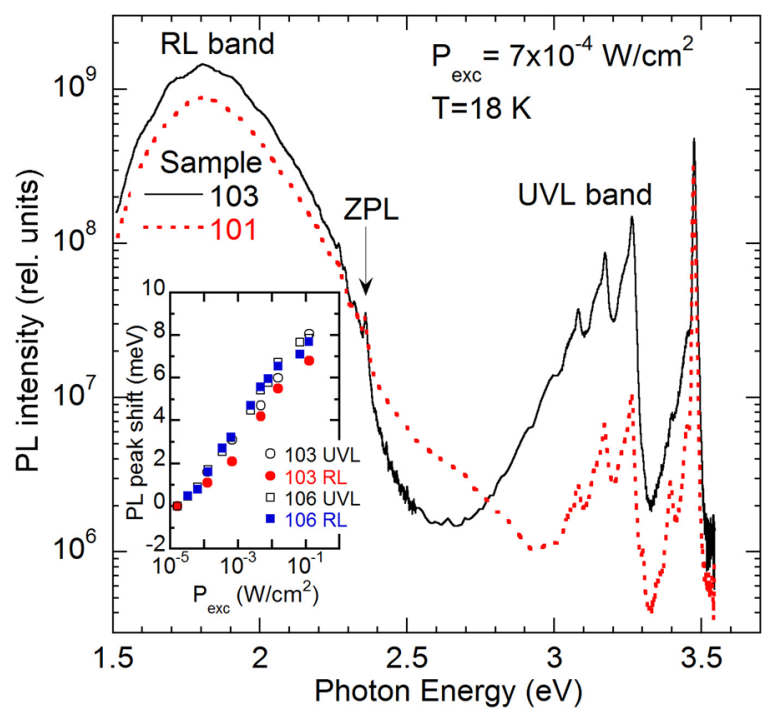

FIG. 2. PL spectra from GaN (samples 101 and 103) at $T=18 \mathrm{~K}$ and $P_{\text {exc }}=7 \times 10^{-4} \mathrm{~W} / \mathrm{cm}^{2}$. The inset shows the shift of the zero-phonon lines with the excitation intensity for the UVL and RL bands in samples.

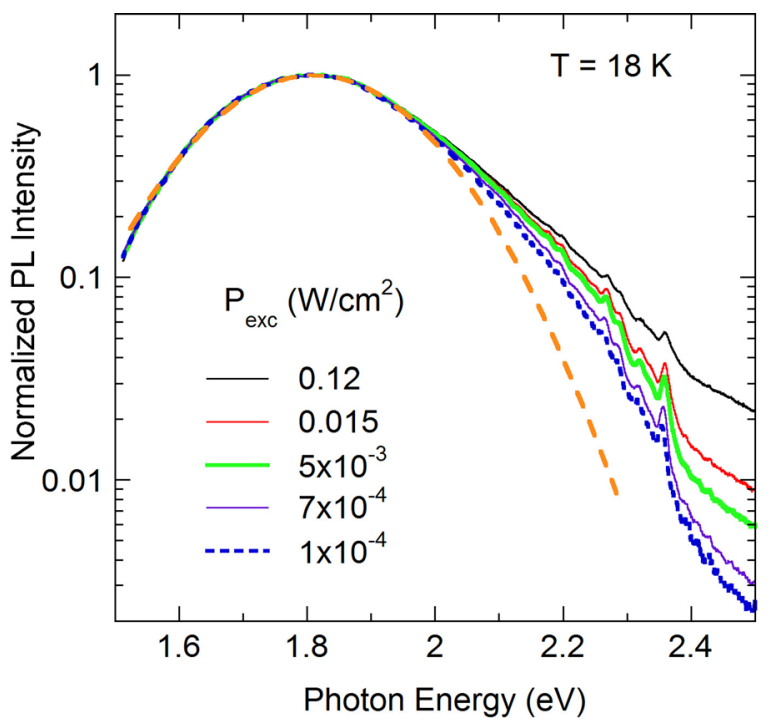

FIG. 3. Low-temperature PL spectra of the RL band (normalized at maximum) at different excitation power densities. The background signal at energies above $2.3 \mathrm{eV}$ is attributed to the GL band having a maximum at $2.5 \mathrm{eV}$. Sample 106. A dashed line is a calculated Gaussian with a maximum at $1.81 \mathrm{eV}$.

Thermal emission of electrons from shallow donors to the conduction band leads to a significant increase of the equilibrium concentration of free electrons (above $10^{14} \mathrm{~cm}^{-3}$ ). As a result, the probability of transitions from the conduction band to the shallow acceptor (which is proportional to $n$ ) increases. The total intensity of the UVL band (eA plus DAP) is nearly independent of temperature. Therefore, the contribution of the DAP transitions decreases with temperature. The separation between the eA and DAP peaks is $19.9 \pm 0.5 \mathrm{meV}$ in all the samples at $40 \mathrm{~K}$. Taking into account the average kinetic energy $(k T / 2)$ of free electrons $(1.7 \mathrm{eV}$ at $40 \mathrm{~K})$ and the ionization energy of the $\mathrm{Si}_{\mathrm{Ga}}$ shallow donors $(30.2 \mathrm{meV}){ }^{11}$ we obtain the effective Coulomb interaction in DAP to be $12 \mathrm{meV}$, which corresponds to the concentration of shallow donors of about $10^{17} \mathrm{~cm}^{-3}$ and is in

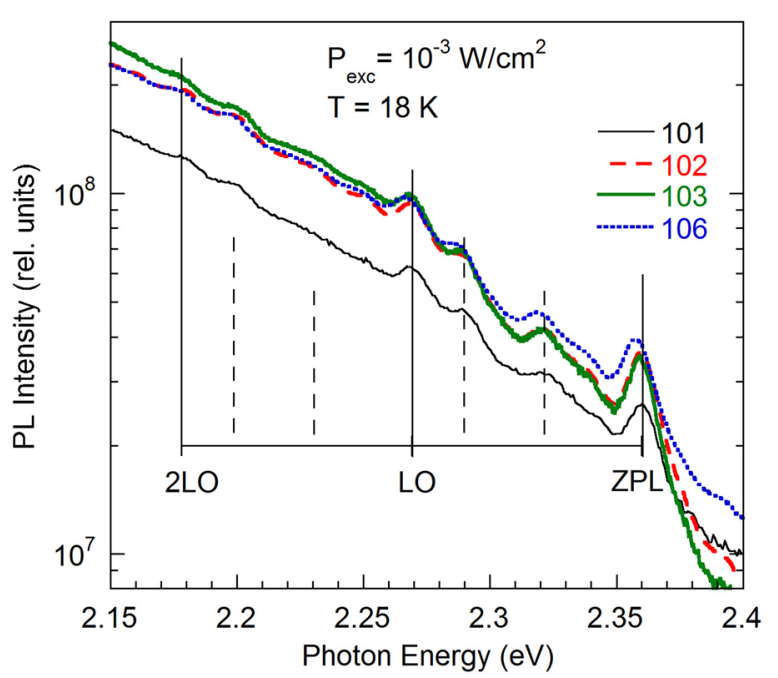

FIG. 4. Fine structure of the RL band in four GaN samples at $T=18 \mathrm{~K}$ and $P_{e x c}=10^{-3} \mathrm{~W} / \mathrm{cm}^{2}$. Vertical solid lines indicate positions of the ZPL line and its LO phonon replicas. Vertical dashed lines indicate positions of phonon replicas corresponding to local or quasi-local phonon modes. 

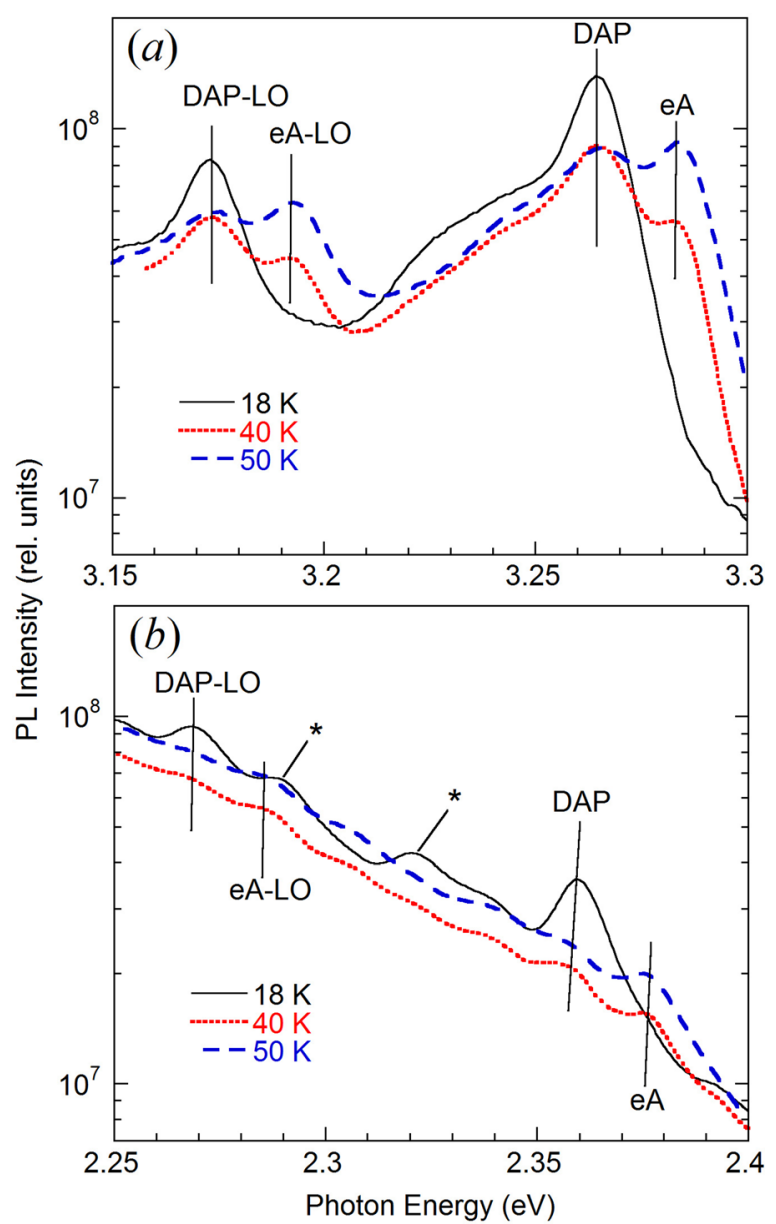

FIG. 5. Evolution of PL spectrum with temperature. (a) High-energy side of the UVL band, (b) high-energy side of the RL band. Vertical lines indicate positions of the eA and DAP peaks and their first LO phonon replicas. Stars indicate local or quasi-local phonon modes.

agreement with the estimate from the time-resolved PL measurements.

A very similar transformation of the PL spectrum with temperature is observed for the high-energy side of the RL band (Fig. 5(b)). The $2.36 \mathrm{eV}$ peak is gradually replaced with the $2.38 \mathrm{eV}$ peak. We assign the peak at $2.36 \mathrm{eV}$ to a nophonon transition of electrons from the shallow donors to a deep acceptor and the $2.38 \mathrm{eV}$ peak to a no-phonon transition of free electrons to the same acceptor. The separation between the DAP and eA peaks is $18.8 \pm 0.5 \mathrm{meV}$ in the studied samples at $40 \mathrm{~K}$. At higher temperatures, the fine structure of the RL band becomes less and less resolved.

An independent proof that the $2.36 \mathrm{eV}$ peak is caused by transitions from a shallow donor to a deep acceptor can be obtained from the peak shift with the excitation intensity. There is a random distribution of the DAP separations, and the dependence of the DAP peak position on the pair separation $R$ is given by ${ }^{12}$

$$
\hbar \omega_{D A P}=E_{g}-E_{D}-E_{A}+\frac{q^{2}}{\varepsilon R}
$$

where $E_{g}$ is the band gap, $E_{D}$ and $E_{A}$ are the donor and acceptor ionization energies, and the last term describes the Coulomb interaction between ionized donors and acceptors with $q$ being the electron charge and $\varepsilon$ being the lowfrequency dielectric constant. For eA transitions,

$$
\hbar \omega_{e A}=E_{g}-E_{D}-E_{A}+\frac{k T}{2}
$$

where the last term accounts for the average kinetic energy of free electrons. With increasing separation in DAP, the PL lifetime becomes longer (due to a smaller overlap of the electron and hole wavefunctions). More distant pairs saturate at lower excitation intensities, and the DAP maximum shifts to higher energies (blue shift) because closer pairs experience stronger Coulomb interaction and contribute at higher photon energies as compared to more distant pairs according to Eq. (1). The relative shifts of the $2.36 \mathrm{eV}$ peak of the RL band and the $3.26 \mathrm{eV}$ peak of the UVL band with variation of the excitation intensity over four orders of magnitude are shown in the inset to Fig. 2. The identical shifts confirm that both peaks have the DAP nature involving the same shallow donor. The maximum shift of the DAP peak is expected to be about $E_{D} / 2 .{ }^{13}$ Note that when deep donors are involved in the DAP transitions, much larger shifts (up to $0.3 \mathrm{eV}$ ) are observed. ${ }^{14}$

The ionization energy of the deep acceptor responsible for the RL band can be calculated from Eq. (2). The position of the DBE peak is between $3.472 \mathrm{eV}$ and $3.476 \mathrm{eV}$ in the studied samples, which is slightly blue-shifted from the value of $3.471 \mathrm{eV}$ in strain-free GaN. ${ }^{15}$ In strain-free $\mathrm{GaN}$, $E_{g}=3.504 \mathrm{eV}$, the value obtained from the PL excitation spectrum at $1.6 \mathrm{~K}$ (Ref. 16) or calculated by taking into account the binding energies of FE and DBE. ${ }^{5}$ Accounting for the sample-dependent strain-induced shift in $E_{g}$ in our samples, we obtain from Eq. (2) that $E_{A}=223 \pm 1 \mathrm{meV}$ for the shallow acceptor involved in the UVL band and $E_{A}=1130 \pm 2 \mathrm{meV}$ for the deep acceptor responsible for the RL band. In these calculations, we used the data for $T=40 \mathrm{~K}$ at which the shrinking of the bandgap is practically compensated by the average kinetic energy of free electrons. In particular, for sample 106, the DAP- and eA-related ZPL lines of the RL band were observed at 2.3557 and $2.3754 \mathrm{eV}$, respectively, and these for the UVL band were found at 3.2606 and $3.2831 \mathrm{eV}$ at $40 \mathrm{~K}$ and excitation intensity of $5 \mathrm{~mW} / \mathrm{cm}^{2}$. From the position of the DBE line at $3.4723 \mathrm{eV}$, we estimate $E_{g}=3.5053 \mathrm{eV}$ for this sample at $18 \mathrm{~K}$. Then, if we ignore the term $k T / 2$ and the variation of $E_{g}$ with temperature in Eq. (2), we obtain $E_{A}=222.2 \mathrm{meV}$ and $E_{A}=1129.9 \mathrm{meV}$ for the shallow and deep acceptors, respectively.

The identity of the shallow and deep acceptors in undoped GaN remains unknown. Previously, we concluded that the shallow acceptor could be $\mathrm{C}_{\mathrm{N}}$ or $\mathrm{Si}_{\mathrm{N}}{ }^{5}$ However, recent calculations have shown that $C_{N}$ is a deep acceptor with an energy level at about $1 \mathrm{eV}$ (Refs. 17 and 18) and $\mathrm{Si}_{\mathrm{N}}$ is an even deeper acceptor with a high formation energy. ${ }^{19}$ The RL band, identical to ours, has been previously associated with the presence of $\mathrm{C}$ and $\mathrm{O}$ (in concentration of about $10^{19} \mathrm{~cm}^{-3}$ for both species) in thick GaN grown by HVPE. ${ }^{8,9}$ These authors observed the RL band from one side of the freestanding $\mathrm{GaN}$ template and attributed it to transitions from a deep donor, $\mathrm{V}_{\mathrm{N}} \mathrm{C}_{\mathrm{N}}$, to a deep acceptor, $\mathrm{V}_{\mathrm{Ga}} \mathrm{O}_{\mathrm{N}}{ }^{9}$ However, our results show that the donor contributing to the 
DAP-type transitions producing the RL band at low temperature is shallow. A similar RL band (maximum at $1.81 \mathrm{eV}$ ) was also observed by Castaldini et $a .^{20}$ in thick HVPE-grown GaN which had low concentration of free electrons. Apparently, the same RL band in undoped HVPE-grown GaN was studied in Refs. 21 and 22. We previously observed the same (but structureless) RL band in high-quality thick or freestanding GaN grown by HVPE at different facilities. ${ }^{5,23,24}$ In all of these works, the fine structure could not be observed, because it was masked by adjacent PL bands (usually the GL band). Note that the red band appearing in GaN grown by molecular beam epitaxy in extremely Ga-rich conditions (labeled in Ref. 5 as the RL2 band) is clearly related to a different defect. In contrast to the RL band, the RL2 band is quenched at temperatures above $100 \mathrm{~K}$ and disappears at about $200 \mathrm{~K}{ }^{5}$ The fact that the RL band is observed in very pure undoped GaN samples $^{23,24}$ indicates that some native defects or the most common contaminations in the HVPE growth are involved in the related defect. Among possible candidates for the defect responsible for RL band, we suggest carbon, silicon, oxygen, and gallium vacancy. Formation of complexes including these defects is also expected.

In summary, we observed fine structure on the highenergy side of the RL band peaking at $1.8 \mathrm{eV}$ in $\mathrm{GaN}$ layers grown by hydride vapor phase epitaxy. The zero-phonon lines at $2.36 \mathrm{eV}$ (at $18 \mathrm{~K}$ ) and $2.38 \mathrm{eV}$ (at $50 \mathrm{~K}$ ) are attributed to transitions from a shallow donor (at low temperature) and from the conduction band (at elevated temperatures), respectively, to a deep acceptor of unknown origin. The transition energy of this acceptor is $1.13 \mathrm{eV}$, and the maximum of the optical transition is at $1.81 \mathrm{eV}$. These accurate experimental data can be used by theorists to improve the theoretical models and to choose the best approximations that later can be applied to other systems.

${ }^{1}$ Y. Saitoh, K. Sumiyoshi, M. Okada, T. Horii, T. Miyazaki, H. Shiomi, M. Ueno, K. Katayama, M. Kiyama, and T. Nakamura, Appl. Phys. Express 3, 081001 (2010).
${ }^{2}$ Y. Wang, H. Xu, S. Alur, Y. Sharma, F. Tong, P. Gartland, T. IssacsSmith, C. Ahyi, J. Williams, M. Park, G. Wheeler, M. Johnson, A. A. Allerman, A. Hanser, T. Paskova, E. A. Preble, and K. R. Evans, Phys. Status Solidi C 8, 2430 (2011).

${ }^{3}$ J. Das, J. Everts, J. van den Keybus, M. Van Hove, D. Visalli, P. Srivastava, D. Marcon, Kai Cheng, M. Leys, S. Decoutere, J. Driesen, and G. Borghs, IEEE Electron Device Lett. 32, 1370 (2011).

${ }^{4}$ M.-W. Ha, C. H. Roh, D. W. Hwang, H. G. Choi, H. J. Song, J. H. Lee, J, H. Park, O. Seok, J. Lim, M.-K. Han, and C.-K. Hahn, Jpn. J. Appl. Phys., Part 1 50, 06GF17 (2011).

${ }^{5}$ M. A. Reshchikov and H. Morkoç, J. Appl. Phys. 97, 061301 (2005).

${ }^{6}$ D. C. Look, C. E. Stutz, R. J. Molnar, K. Saarinen, and Z. Liliental-Weber, Solid State Commun. 117, 571 (2001).

${ }^{7}$ J. D. McNamara, M. Foussekis, H. Liu, H. Morkoç, M. A. Reshchikov, and A. A. Baski, Proc. SPIE 8262, 826213 (2012).

${ }^{8}$ L. Wang, U. Zeimer, E. Richter, M. Herms, and M. Weyers, Phys. Status Solidi A 203, 1663 (2006).

${ }^{9}$ L. Wang, E. Richter, and M. Weyers, Phys. Status Solidi A 204, 846 (2007).

${ }^{10}$ M. A. Reshchikov, D. Huang, F. Yun, L. He, H. Morkoç, D. C. Reynolds, S. S. Park, and K. Y. Lee, Appl. Phys. Lett. 79, 3779 (2001).

${ }^{11}$ W. J. Moore, J. A. Freitas, Jr., S. K. Lee, S. S. Park, and J. Y. Han, Phys. Rev. B 65, 081201 (2002).

${ }^{12}$ D. G. Thomas, J. J. Hopfield, and W. M. Augustyniak, Phys. Rev. 140, A202 (1965).

${ }^{13}$ E. Zacks and A. Halperin, Phys. Rev. B 6, 3072 (1972).

${ }^{14}$ M. A. Reshchikov, G.-C. Yi, and B. W. Wessels, Phys. Rev. B 59, 13176 (1999).

${ }^{15}$ K. Kornitzer, T. Ebner, K. Thonke, R. Sauer, C. Kirchner, V. Schwegler, M. Kamp, M. Leszczynski, I. Grzegory, and S. Porowski, Phys. Rev. B 60, 1471 (1999).

${ }^{16}$ B. Monemar, Phys. Rev. B 10, 676 (1974).

${ }^{17}$ J. L. Lyons, A. Janotti, and C. G. Van de Walle, Appl. Phys. Lett. 97, 152108 (2010).

${ }^{18}$ D. O. Demchenko, I. C. Diallo, and M. A. Reshchikov, Phys. Rev. Lett. 110, 087404 (2013).

${ }^{19}$ D. O. Demchenko, private communication (2013).

${ }^{20}$ A. Castaldini, A. Cavallini, L. Polenta, C. Diaz-Guerra, and J. Piqueras, J. Phys.: Condens. Matter 14, 13095 (2002).

${ }^{21}$ W. Götz, L. T. Romano, B. S. Krusor, N. M. Johnson, and R. J. Molnar, Appl. Phys. Lett. 69, 242 (1996).

${ }^{22}$ C. Bozdog, H. Przybylinska, G. D. Watkins, V. Härle, F. Scholz, M. Nayer, M. Kamp, R. J. Molnar, A. E. Wickenden, D. D. Koleske, and R. L. Henry, Phys. Rev. B 59, 12479 (1999).

${ }^{23}$ M. A. Reshchikov, M. Zafar Iqbal, S. S. Park, K. Y. Lee, D. Tsvetkov, V. Dmitriev, and H. Morkoç, Physica B 340-342, 444 (2003).

${ }^{24}$ J. D. McNamara, M. A. Foussekis, A. A. Baski, X. Li, V. Avrutin, H. Morkoç, J. H. Leach, T. Paskova, K. Udwary, E. Preble, and M. A. Reshchikov, Phys. Status Solidi C 10, 536 (2013). 